\title{
PROCESS PARAMETER OPTIMIZATION OF LAP JOINT FILLET WELD BASED ON FEM- RSM-GA INTEGRATION TECHNIQUE
}

M. Islam ${ }^{\mathrm{a}, \mathrm{b},{ }^{*}}$, A.Buijk $^{\mathrm{c}}$, M. Rais-Rohani ${ }^{\mathrm{b}, \mathrm{d}}$, K. Motoyama ${ }^{\mathrm{b}, \mathrm{d}}$

${ }^{a}$ Ahsanullah University of Science and Technology, Dhaka, 1205, Bangladesh

${ }^{\mathrm{b}}$ Center for Advanced Vehicular Systems, Mississippi State University, MS 39762, USA

${ }^{c}$ Simufact-Americas LLC, Plymouth, MI 48170-4347,USA

${ }^{\mathrm{d}}$ Department of Aerospace Engineering, Mississippi State University, MS 39762, USA

\begin{abstract}
This study introduces a welding process design tool to determine optimal arc welding process parameters based on Finite Element Method (FEM), Response Surface Method (RSM) and Genetic Algorithms (GA). Here, a sequentially integrated FEM-RSM-GA framework has been developed and implemented to reduce the weld induced distortion in the final welded structure. It efficiently incorporates finite element based numerical welding simulations to investigate the desired responses and the effect of design variables without expensive trial experiments. To demonstrate the effectiveness of the proposed methodology, a lap joint fillet weld specimen has been used in this paper. Four process parameters namely arc voltage, input current, welding speed and welding direction have been optimized to minimize the distortion of the structure. The optimization results revealed the effectiveness of the methodology for welding process design with reduced cost and time.
\end{abstract}

Keywords: Welding simulation, finite element modeling, process optimization, genetic algorithms, response surface method.

\section{Introduction}

Arc welding is a popular metal joining process that finds wide range of applications in various manufacturing industries especially automotive, shipbuilding, and aerospace industries. Its major advantages are reduced cost, enhanced joint strength, and efficiency. However, welding induces a permanent deformation known as weld induced distortion in the final welded product. Distortion has critical negative impact on the quality of the welded product especially on joint strength and dimensional accuracy. Therefore, expensive and time consuming post-weld treatments are required to achieve desired level of dimensional accuracy of the welded products. For applications where cost control is critical, the goal is to keep the distortion level as low as possible.

Several parameters in the welding procedure contribute to the distortion. These include heat input, welding sequence, degree of restraint, welding conditions and joint details. Better control of these welding variables will eliminate the conditions that promote distortion (Tsai et al., 1999). Several researchers investigated experimental process parameter optimization techniques for arc welding process. In experimental optimization, a mathematical model is developed correlating the process parameters with desired objective function using statistical techniques such as Design of Experiments (DOE), Taguchi method, Response Surface Methodology (RSM) and so on. Next, the developed mathematical model is executed in optimization algorithm for parametric optimization. Evolutionary Algorithms and Artificial Neural Networks are the most frequently used optimization methods for this purpose. Benyounis et al. (Benyounis and Olabi, 2008) and Dhas et al. (a and S, 2012) provided a detailed review of experimental optimization of welding process parameters. 
Sudhakaran et al. (Sudhakaran et al., 2012) investigated optimization of process parameters using particle swarm optimization to minimize angular distortion in 202 grade stainless steel gas tungsten arc welded plates. Correia et al. (Correia et al., 2004) investigated the implementation possibility of using GA as a method to decide near optimal settings of a GMAW process and compared its performance with RSM. Kim et al. (Kim et al., 2002) suggested a GA and RSM combined approach for determining optimal welding conditions. Later, Kim et al. (Kim et al., 2005) have proposed a method for determining the nearoptimal setting of GMAW process parameters using a controlled random search (CRS).

Experiment based process parameter optimization is by far the most popular technique for welding. However, it is often a very costly and time consuming approach due to many kinds of nonlinear events involved (Correia et al., 2005). Numerical welding simulations can help in this regard. For more than three decades, Finite Element Method (FEM) has been the most popular and powerful tool for simulating the thermo-mechanical behavior of a structures during welding process. Lindgren has provided a detailed description on computational modeling of welding process in his papers (Lindgren, 2001a, b; Lingren, 2001). Many researchers successfully used FEM for welding simulation especially for residual stress and weld induced distortion prediction. The initial welding simulations were highly simplified based on twodimensional (2D) approach and plane strain condition (Lindgren, 2001a). Although the 2D analysis gives indications of the welding residual stresses involved in quasi-static, plane strain conditions, it does not provide the total out-of-plane deformations (Camilleri and Gray, 2005). Brown and Song (Brown and Song, 1992) used both two-dimensional (2D) and three-dimensional (3D) models to investigate fixturing impact on large structures and concluded that full 3D models are essential in predicting welding distortion. Michaleris et al. (Michaleris and Debiccari, 1996, 1997) used two step numerical analysis approach that combines 2D welding simulation with 3D structural analysis for predicting buckling distortion; 2D welding simulation substantially reduces the computation time of their analysis. Tsai et al. (Tsai et al., 1999) investigated distortion mechanism and the effect of welding sequence on panel distortion using FEM based on inherent shrinkage method. Deng et al. (Deng et al., 2007a; Deng and Murakawa, 2008a, b; Deng et al., 2007b; Deng et al., 2008; Deng et al., 2010) conducted substantial research on predicting welding distortion of welded structures.

It is evident that FEM is a very useful and reliable welding process modeling and analysis tool. However, FEM weld models still contribute little to the development of modern welding technology. One of the major application fields of welding simulations can be process parameter optimization because it can effectively replace the expensive experiments. Very few research works have been conducted in this domain. Kadivar et al. (Kadivar et al., 2000) linked Genetic Algorithms (GA) with a transient 2D FE model to determine optimum welding sequence of a circular pipe for minimization of distortion. Song et al. (Song et al., 2003; Song et al., 2004) investigated sensitivity of thermo-mechanical responses of welded joints to variation in material properties and optimized quasi-static weakly coupled thermo-elastoplastic FE process for side heater design. Islam et al. (Islam et al., 2014) (Islam et al., 2013) developed a direct computational framework based on integrated computational tools, which allows automatic optimization of welding process parameters without the requirement of expensive experiments.

In this paper, an innovative FEM-RSM-GA integrated approach for process parameter optimization is proposed. The proposed approach essentially mimics the experimental optimization procedure except it incorporates finite element (FE) models in lieu of expensive experiments for required analysis. First, a 3D thermo-elastic-plastic FE model is developed and validated by experimental results. RSM is then applied for Design of Experiments and analyzing the responses of interest by developing required mathematical models. GA, for its wide acceptance for process optimization, is subsequently implemented to determine optimum process control parameters. To demonstrate the effectiveness of the proposed optimization approach, a lap joint specimen has been investigated. Optimization of process parameters has been carried out to minimize the weld induced distortion in the final welded structure. Total weld induced distortion is set as objective function (that will be minimized) and minimum weld quality requirement in the form of 
sufficient weld fusion or melting is set as optimization constraint. The important parameters including welding speed, input current, arc voltage and welding direction or sequence, etc. are treated as design variables during the analysis.

The remainder of the paper is organized as follows. In next section, the general framework of the proposed FEM-RSM-GA integrated optimization approach is discussed and then FE based welding simulation and model validation approach has been briefly introduced. In Section 4, experimental design and response surface modeling strategy have been discussed in detail. Finally, genetic algorithm based optimization problem formulation and optimization results have been summarized in Section 5.

\section{General Framework of Integration System}

The proposed FEM-RSM-GA approach is based on three different sequentially coupled stages as illustrated in Figure 1. The first stage consists in setting up the numerical simulation of considered welding process problem through finite element modeling. The welding simulation tool chosen for this research work is simufact.welding $($, which is a Pre- and Postprocessor-GUI for FE welding process analysis. There are several fundamental reasons for the selection of this FEM package, foremost among which are its ability to simulate complex welding process model with multiple welding robots working at the same time or different times, flexibility to define or modify welding parameters, paths, directions and simple automatic batch running option useful for simulation-based design optimization. Simufact also provides a user-friendly interface for configuring process modeling properties, solver settings, welding robot settings, heat source parameters and geometric boundary conditions.

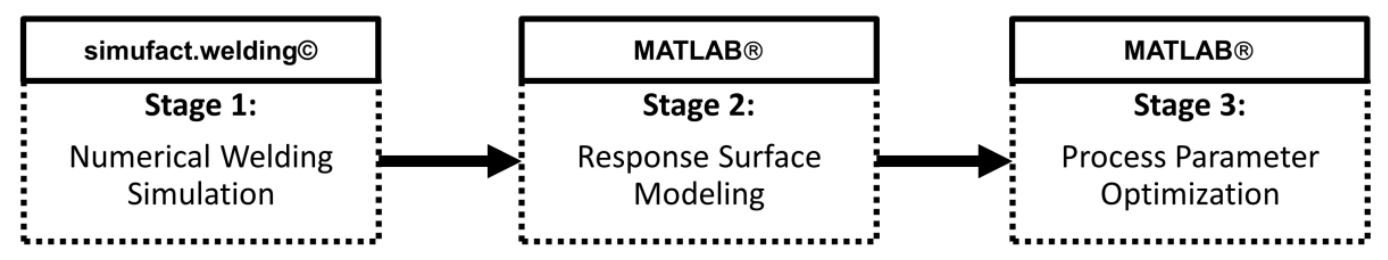

Figure 1. The framework of FEM-RSM-GA system.

The second stage is focused on Design of Experiments and development of response surface model. This stage consists of following four steps-

- Systematic design of a series of experiments and generation of the response data,

- Selection of a suitable RSM model to adequately represent the data,

- Training and fitting the mathematical model with measured data,

- Validation of the model by measuring different accuracy or error parameters.

Simulation model developed and validated in stage one is used here for conducting the experiments and recording required response data. Next, a quadratic response surface model is fitted to the obtained data. A customized computer program is developed in the MATLAB programming environment to facilitate the development and performance analysis of the model. During this stage, it was ensured the developed mathematical model represents an adequate and reliable correlation between the design variables (parameters to be optimized) and considered objectives (criterions of optimizations). In this stage, a DOE table is constructed first using the popular Box-Behnken method, and subsequently the response at each design point of the DOE table is evaluated by the welding simulation. After calculating the response values at all the DOE points, a quadratic PRS is developed using the least squares technique. Accuracy of the response surface model largely depends on the nonlinearity of the system, the number and range of design points in the DOE table. The PRS model is not guaranteed to be adequately accurate in the first 
trial. As such, an iterative process of DOE table construction-PRS fitting-error checking is carried out to fit the PRS with adequate accuracy within the design space defined by the DOE table.

The third stage is concerned with the process parameter optimization based on Response Surface Model. Genetic Algorithms (GA) has been used as optimization method as it is well known to be efficient and to converge to global optimum point specially for manufacturing process related problems. MATLAB ${ }^{\circledR}$ GA solver is used as the optimization tool. A customized GA variant is developed by varying different default solver properties to meet the problem-specific requirements. The PRS is linked with GA for design optimization in this stage. The overall optimization process based on FEM-RSM-GA integrated technique is depicted in the flowchart of Figure 2. Details of each of the three stages are discussed in later sections of this paper.

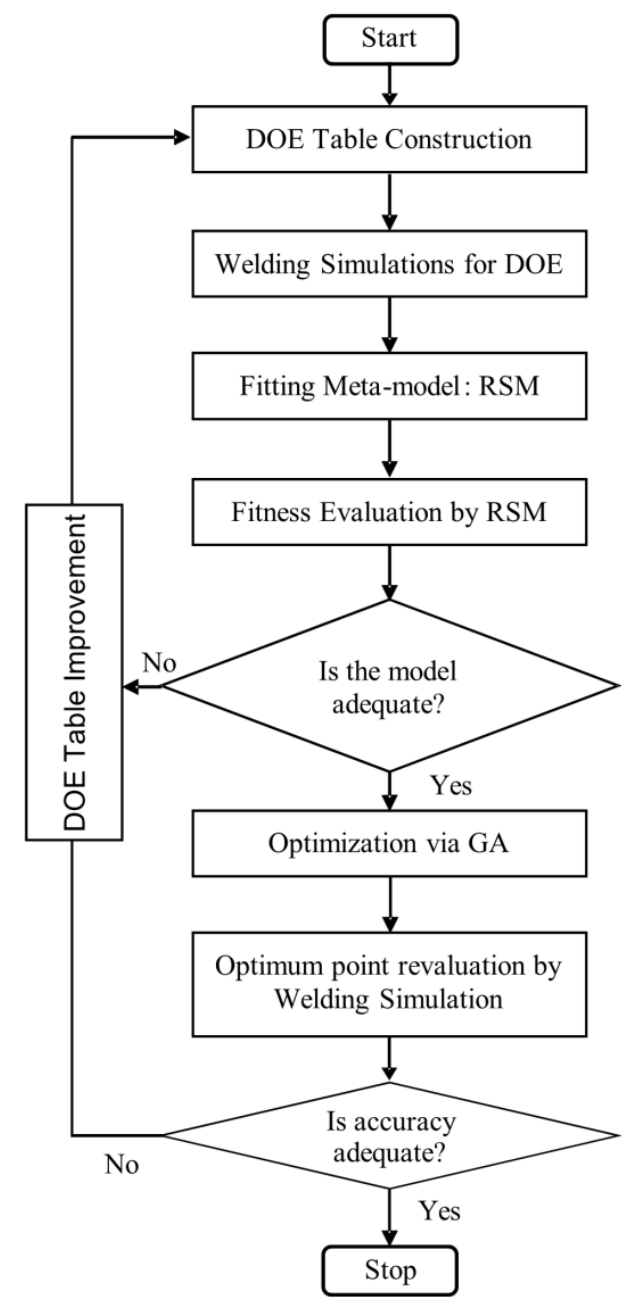

Figure 2. A flowchart of FEM-RSM-GA integrated optimization process.

\section{Welding Simulation Approach}

\subsection{Problem Definition}

The problem we are considering to demonstrate the implementation of our optimization system is a simple lap joint of two plates at the middle section of the plates. All the dimensions of the specimen have been illustrated in Figure 3. The material of the plates is ASTM A591M-89 sheet metal steel. The total 
weld length is $70 \mathrm{~mm}$ and four clamps have been used to restrain the movement of the plates during welding process. The corresponding three dimensional FE model has been illustrated in Figure 3 .

\subsection{Thermo-mechanical Analysis}

Computational modeling of welding is a coupled thermo-mechanical problem. It involves several coupled phenomena such as complex weld pool physics, nonlinear heat flow, nonlinear material behavior at elevated temperature, generation of thermal stresses and mechanical deformations etc. Lindgren (Lindgren, 2007) and Goldak (Goldak and Akhlaghi, 2005) have provided an extensive review on different interactions present in welding process and their impact on numerical modeling of welding. However, the main stream in computational welding mechanics (CWM) is the use of weakly coupled models where the physics in the weld is replaced by a simplified heat input model and thermal analysis is performed independent of mechanical analysis. Such approach is useful and computationally efficient when the primary objective is to investigate transient temperature, deformation and stress field other than complex metal physics properties. In this paper, since our objective is to investigate weld induced distortion in the welded structure, weakly coupled modeling approach has been adopted for welding simulation.
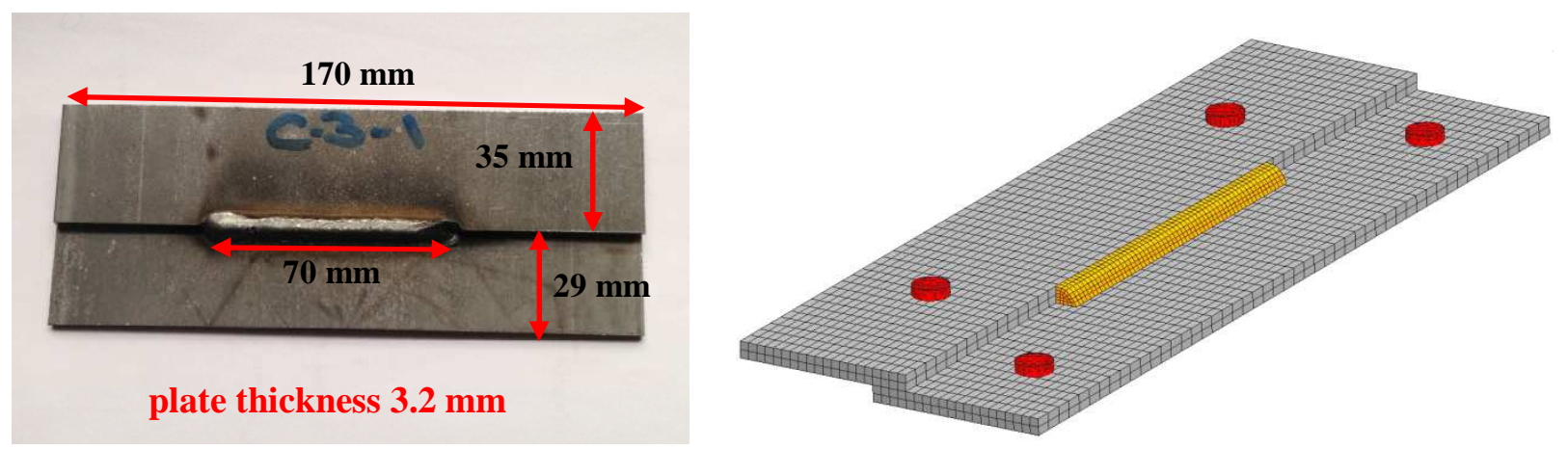

Figure 3. Experimental model (left) and 3D FE model (right) of lap joint specimen.

Three-dimensional thermo-mechanical FE simulations were carried out using Marc solver of simufact.welding $\odot$. Marc uses a staggered solution procedure in coupled thermo-mechanical analysis, where it first performs a thermal analysis, then mechanical analysis. An automatic local mesh refinement has been implemented to improve simulation result accuracy while not increasing the computational time considerably. As such, the focus is not directed on the meshing the entire model but on the areas of welding zones. Each refinement level halves the element size in the heat input area. A refinement level of two was used in this analysis which results in quartering the element length in the vicinity of the weld zone. Heat source area is treated as refinement criterion and the criterion is set by means of a scaling factor (equal to 2), which is a multiplier of the heat source size for the local refinement area around the heat source. Figure 4 illustrates the locally refined mesh in the vicinity of the weld bead during analysis.

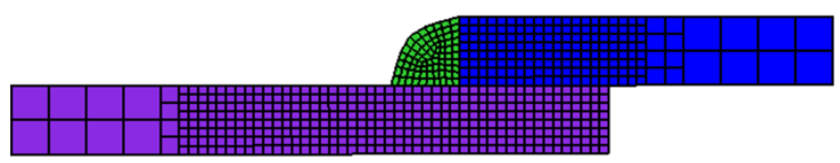

Figure 4. Local mesh refinement in the vicinity of the weld bead.

The classical heat conduction formulation using temperature dependent material properties and a moving heat source constitutes the thermal problem that needs to be solved to obtain temperature history at each node of the FE model. The popular double ellipsoid heat source model, first proposed by Goldak et al 
(Goldak et al., 1984), is used to simulate the arc welding heat input. It is a non-axisymmetric heat source where the front half of the source is the quadrant of one ellipsoid and the rear half is the quadrant of another ellipsoid. The Gaussian heat flux distribution has been implemented along the longitudinal axis of the heat source model. Heat losses due to convection, radiation and contact have been considered by taking into account the corresponding thermal coefficients.

In mechanical analysis, the basic equations are the equilibrium equations, constitutive stress-strain relations and geometric compatibility equations (Lindgren, 2007). Temperature history obtained from thermal analysis is input as thermal loading into the structural model to calculate the stress-strain field. The change in the temperature distribution contributes to the deformation of the body through thermal strains and influences the material properties. Large deformation and large plastic strains are accounted for and the additive decomposition of elastic, plastic and thermal strain contributions is utilized for the stress recovery process. The analysis uses a regular elasto-plastic material model with von Mises yield criterion. Phase transformation effects are not considered in the current analysis.

\subsection{Model Validation}

The heat source and out-of-plane distortion predictions of the present FE model of lap joint specimen have been validated by experimental results. Physically, the width and depth of heat source correspond to the weld bead cross-section dimensions. As such, the heat source model was validated with respect to the weld macrograph of experimental weld cross-sections and a fairly good agreement was achieved in terms of weld pool boundary shape and size as shown in Figure 4. Experimentally obtained weld pool shape is shown in Figure 5 (a) by yellow dotted line and the corresponding simulation prediction of weld pool shape has been depicted in Figure 5 (b) with the black dotted line.

(a)
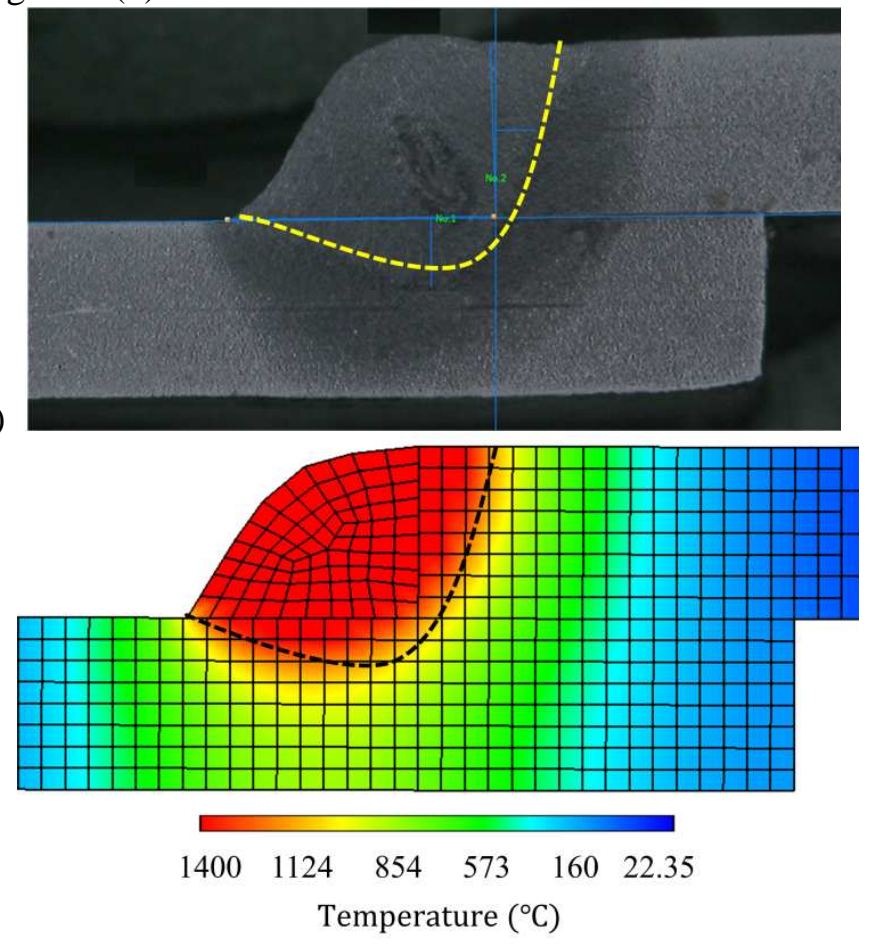

Figure 5. Weld pool shape comparison- (a) experimental and (b) simulation prediction.

After validating the heat source, the simulation predicted out-of-plane distortion pattern was compared with the experimental results as shown in Figure 6. Experimental out-of-plane distortion pattern is illustrated in Figure 6 (a) which indicates the upper plate is distorted into a convex shape after complete 
cool down where its high ends are separated from the lower plate. On the other hand, the lower plate is distorted into a concave shape where its middle section towards edge has moved up after complete cool down. Out-of-plane distortions at left and right edges of the upper plate were around $0.1 \mathrm{~mm}$ and $0.4 \mathrm{~mm}$ respectively. Moreover, the maximum distortion occurred at the middle section of the lower plate which is around $0.6 \mathrm{~mm}$. The corresponding simulation predicted out-of-plane distortion pattern has been shown in Figure 6 (b) and the contour plot of z-distortion indicates good agreement with experimental results as well. The simulation prediction is scaled two times for better visualization purpose. Readers are referred to paper (Islam et al., 2014; Islam et al., 2013) for details of FE modeling and validation of this model.
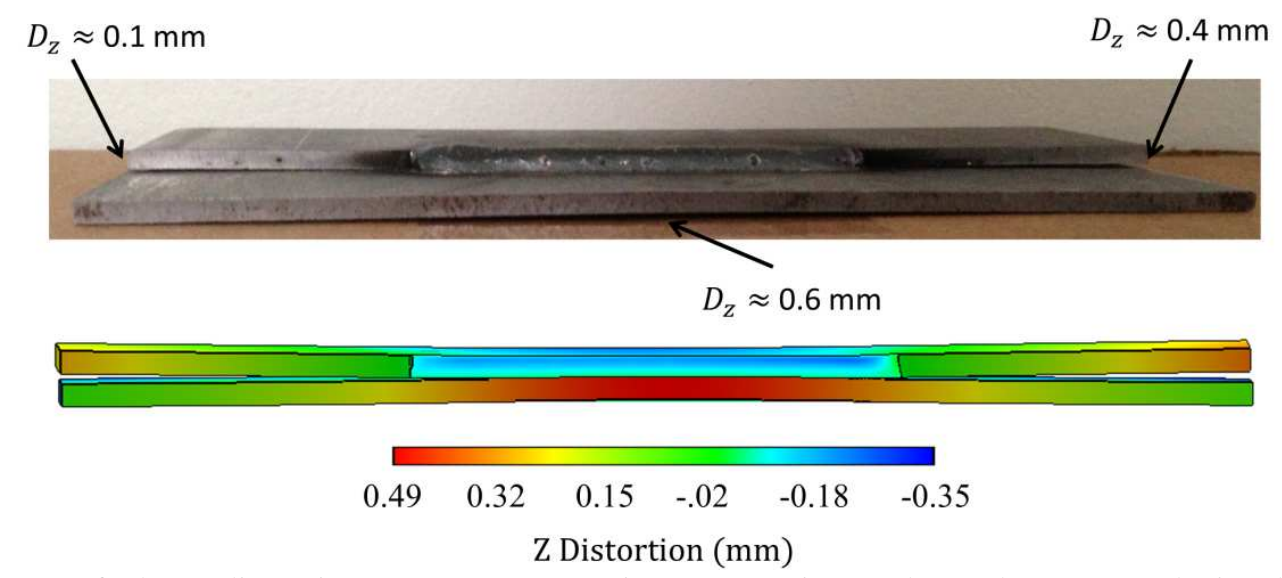

Figure 6. Out-of-plane distortion pattern comparison- experimental result (top) and simulation result (bottom).

\section{Design of Experiments and Response Surface Modeling}

\subsection{Overview of Response Surface Methodology}

RSM is a collection of statistical and mathematical techniques useful for developing, improving and optimizing processes (Montgomery, 1997). Despite the steady growth in computing power, the complexity and high cost of FEA seem to keep pace with computing advancement (Park and Dang, 2010). As such, it may not be feasible to conduct iterative optimization directly using computation intensive simulations for objective function evaluations as this may greatly increase the overall design cost. The common approach to deal with this problem is to carry out a number of computer simulations based on Design of Experiments (DOE) method and develop an inexpensive response surface model approximating the relationship between input variables and desired responses. RSM approximates the unknown functional relationship between a response of interest and input variables by a polynomial response surface (PRS). The quadratic polynomial model is the most popular PRS because of its flexibility to take on a wide variety of functional forms, ease of estimating the parameters and several practical success experiences in solving real engineering problems. The mathematical model of quadratic response surface is described as

$$
y=\beta_{o}+\sum_{i=1}^{k} \beta_{i} x_{i}+\sum_{i=1}^{k} \beta_{i i} x_{i}^{2}+\sum_{i=1}^{k-1} \sum_{j=k+1}^{k} \beta_{i j} x_{i} x_{j}+\epsilon
$$

where $\mathrm{k}$ is the number of variables, $\varepsilon$ is the error and $\beta \mathrm{s}$ are the constant regression coefficients. The quadratic model in Eq. (4.1) includes $p=(k+1)(k+2) / 2$ unknown coefficients $\left(\beta_{0}, \beta_{i}, \beta_{i i}\right.$, and $\left.\beta_{i j}\right)$ that are found using the least squares technique based on the true response values at a set of $n \geq p$ training points. Various DOE techniques such as Latin Hypercube Sample (LHS), Taguchi orthogonal arrays, Central Composite Design (CCD) and Box-Behenden Design may be used for identifying the training set in the 
design space bounded by the lower and upper bounds of $x_{i}, i=1, k$. The accuracy of a surrogate model is dependent on the number of training points and how these training points are distributed in the design space. For a PRS, at least $3 k$ training points are necessary to build a good surrogate model (Montgomery, 1997).

\subsection{Sensitivity Analysis}

A two-level DOE-based sensitivity analysis is conducted as a first step to investigate the sensitivity of the chosen output (max weld induced distortion) to the selected design variables (current, voltage, speed and direction). The arbitrarily selected baseline point and its corresponding response are given in Table 1. Since welding direction is not a continuous variable, it is difficult to interpret the response of the output to the change in welding direction. Therefore, a separate sensitivity analysis was carried out with the other three design variables for arbitrarily selected welding direction (i.e., X4 value). Physical meaning of each welding direction is described in Table 5. The difference of the highest relative to the lowest max distortion value in Table 1 is approximately $25 \%$.

Table 1. Baseline design variable values and corresponding response value.

\begin{tabular}{|c|c|c|c|c|}
\hline $\begin{array}{l}\text { Current } \\
\text { (X1) } \\
\text { Ampere }\end{array}$ & $\begin{array}{c}\text { Voltage } \\
\text { (X2) } \\
\text { Volt }\end{array}$ & $\begin{array}{l}\text { Speed } \\
(\mathrm{X} 3) \\
\mathrm{mm} / \mathrm{s}\end{array}$ & $\begin{array}{l}\text { Direction } \\
\text { (X4) }\end{array}$ & $\begin{array}{c}\text { Max } \\
\text { Distortion, } \\
\mathrm{mm}\end{array}$ \\
\hline 150 & 15 & 7 & 1 & 0.523 \\
\hline 150 & 15 & 7 & 2 & 0.512 \\
\hline 150 & 15 & 7 & 4 & 0.584 \\
\hline 150 & 15 & 7 & 6 & 0.641 \\
\hline
\end{tabular}

The sensitivity analysis was formulated such that each variable can take either a high or a low value for each design point while the other two variables are kept at their respective baseline values. As such, the calculated sensitivity represents the percentile main effect of each design variable on the max weld induced distortion. The high and low values for each design variable were selected as $+/-33.33 \%$ of their baseline value. Figure 7 presents the percentile main effect of each design variable on the max welding induced distortion for four arbitrary welding directions. Blue color indicates the corresponding design variable has been set at [baseline - 33.33\%] value and it is termed as low value. On the other hand, the maroon color indicates the corresponding design variable has been set at [baseline $+33.33 \%$ ] value and it is termed as high value. 

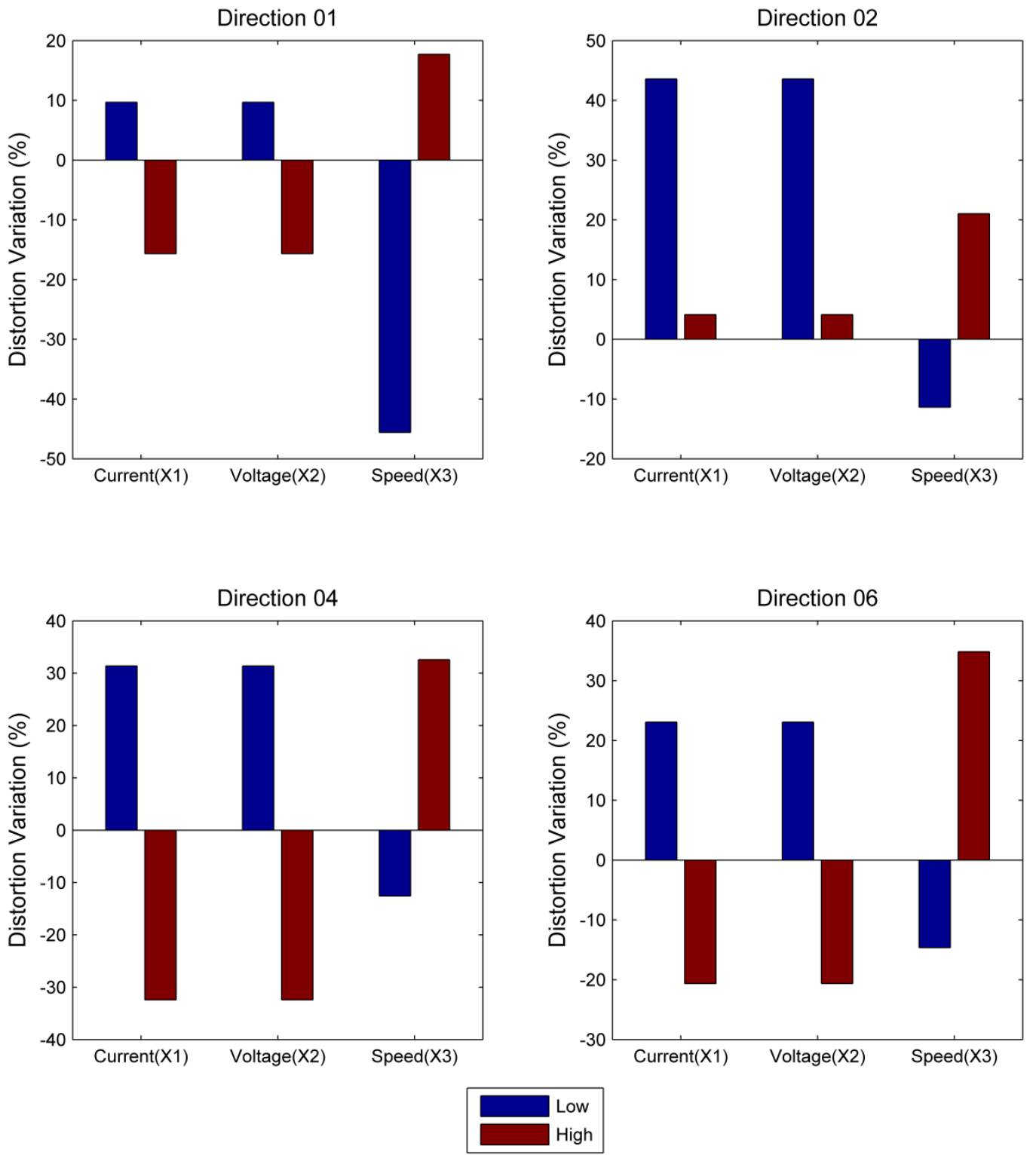

Figure 7. Percentile main effects of design variables on maximum distortion for different directions.

It can be seen through the investigation of the sensitivity results that the main effects of current, voltage and speed on distortion are highly dependent on welding direction for the lap-joint model. Current and voltage have less effect on distortion than welding speed for welding direction 1 but more effect for welding direction 2. For example, decrement of current from baseline value to low value (blue column) causes maximum distortion increment of $9.68 \%$ and $43.57 \%$ for welding direction 1 and welding direction 2 respectively. Increment of voltage from baseline value to high value (maroon column) reduces maximum distortion by $15.7 \%$ for direction 1 . However, same adjustment of voltage from baseline value to high value (maroon column) actually increases maximum distortion by $4.14 \%$ for direction 2 . Again, same adjustment of voltage reduces maximum distortion by $32.43 \%$ and $20.63 \%$ respectively for welding direction 4 and direction 6 . 


\subsection{Experimental Design}

Experimental design was created based on a four factor three-level Box-Behnken method. Two different DOE tables has been generated. One is for modeling welding induced distortion and the other one is for temperature constraint. Consideration of temperature constraint is important in order to ensure sufficient weld quality in addition to minimize the distortion. Details of temperature constraint consideration has been discussed in the optimization section of this paper. For distortion predicting RSM, separate DOE tables with three design variables was constructed for each welding direction since welding direction (X4) is a discrete design variable. Subsequently, all DOE tables were merged to formulate the final DOE table for all four variables including the welding direction. Table 2 shows the experimental design labels of considered variables.

Table 2. Experimental design labels of variables.

\begin{tabular}{ccccc}
\hline Design Variable & Symbol & -1 & 0 & 1 \\
\hline Current (Ampere) & X1 & 100 & 150 & 250 \\
Voltage (Volt) & X2 & 10 & 15 & 25 \\
Welding Speed (mm/s) & X3 & 3.5 & 7 & 10 \\
\hline
\end{tabular}

A second degree polynomial was fitted to the DOE table data and the adequacy of the fitted PRS for distortion prediction was measured by three different error statistics (i.e., average error, maximum error and Root Mean Square (RMS) error). A trial-error-modification loop based methodology was adopted to improve the fitting of the PRS to the experimental data until the absolute maximum error was 5\% or less. It took six trials to reduce the maximum fitting error below $5 \%$ as shown in Table 3.

Table 3. Absolute error analysis results of distortion PRS.

\begin{tabular}{llll}
\hline \multirow{2}{*}{ Trial \# } & $\begin{array}{l}\text { Average } \\
\text { Error(\%) }\end{array}$ & $\begin{array}{l}\text { Maximum } \\
\text { Error }(\%)\end{array}$ & $\begin{array}{l}\text { RMS } \\
\text { Error }(\%)\end{array}$ \\
\hline 1 & 3.19 & 10.64 & 4.52 \\
2 & 2.49 & 9.4 & 3.48 \\
3 & 2.23 & 6.94 & 3.11 \\
4 & 1.92 & 6.16 & 2.69 \\
5 & 1.76 & 5.39 & 2.46 \\
6 & 1.74 & 4.36 & 2.34 \\
\hline
\end{tabular}

Figure 8 (a) shows the relationship between actual and PRS predicted max distortion values. It is evident from the figure that the model is sufficiently accurate as the predictions are placed within acceptable tolerance limit from the diagonal line of actual value. 

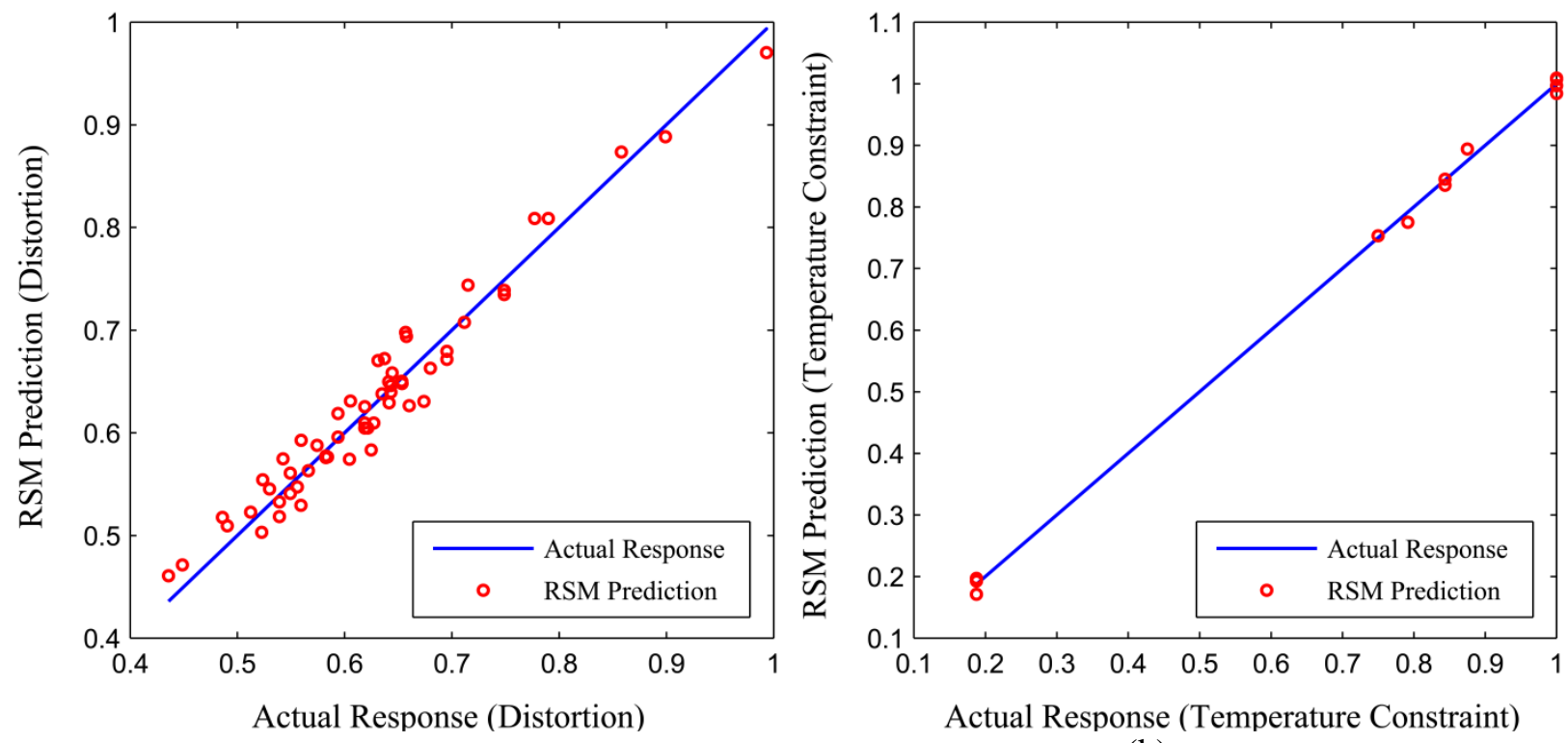

Figure 8. Fitness plots of (a) distortion RSM and (b) temperature constraint RSM.

Similarly, a full quadratic response surface model was generated to replace the computation intensive FE simulation during optimization for temperature constraint evaluation. Since temperature constraint is independent of welding direction, only welding current, arc voltage and speed are considered as design variables. Experimental design was created based on a three-level Box-Behnken method with 15 data points and experimental design levels were the same as that shown in Table 1. A second degree polynomial was fitted to the experimental data and the PRS with adequate fitting accuracy was obtained with just one trial. As shown in Figure 8(b), a temperature constraint value of 1 indicates that the constraint is satisfied or not violated. Physically, satisfaction of temperature constraint indicates that the heat input was sufficient to ensure required weld penetration.

\subsection{Effect of Process Parameters on Distortion}

The mathematical model developed to correlate the weld induced distortion with welding process variables can be used to investigate parametric effect of considered variables on distortion. Analysis of direct and interaction effects of process variables have been represented in graphical form in Figure 9-11.

Figure 9 illustrates the direct effect of individual parameters on weld induced distortion. In order to accommodate the effect of all variables in a single plot, the design variables have been scaled with respect of their upper bound. As shown in the figure, distortion increases with increase in both arc voltage and input current. It is obvious because with increase of arc voltage and input current, the heat input also increases as well which ultimately results in higher temperature, stress and distortion. Furthermore, it is seen that an increase in welding speed decreases the distortion because the net heat input at a given time decreases with speed. As such, a high welding speed is always desirable to keep the distortion level as low as possible. However, a very high speed often causes poor weld penetration resulting in negative effect on weld strength and quality. Therefore, welding speed is required to be optimized to minimize distortion while ensuring sufficient weld quality. 
Figure 9. Direct of scaled design variables on weld induced distortion. [The variables are scaled with respect to their upper bound as shown in Table 4].

The interaction effect of voltage and speed on distortion has been investigated by contour plots as shown in Figure 10. Three contour surfaces have been generated to demonstrate the interaction effect for three different levels $(+1$, base and -1 level) of other two variables namely input current and welding direction. A coarse, medium and fine mesh size have been used in the figure to distinguish the $+1,0$ and -1 levels of other two variables respectively. As illustrated in Figure 10, with increase in arc voltage the distortion also increases while it decreases with increase in welding speed. Thereby, the optimum point is likely to be located at a region where the arc voltage is at near lower bound while welding speed is at near upper bound. Also, it is observed that distortion value also increases with increase in other two variables.

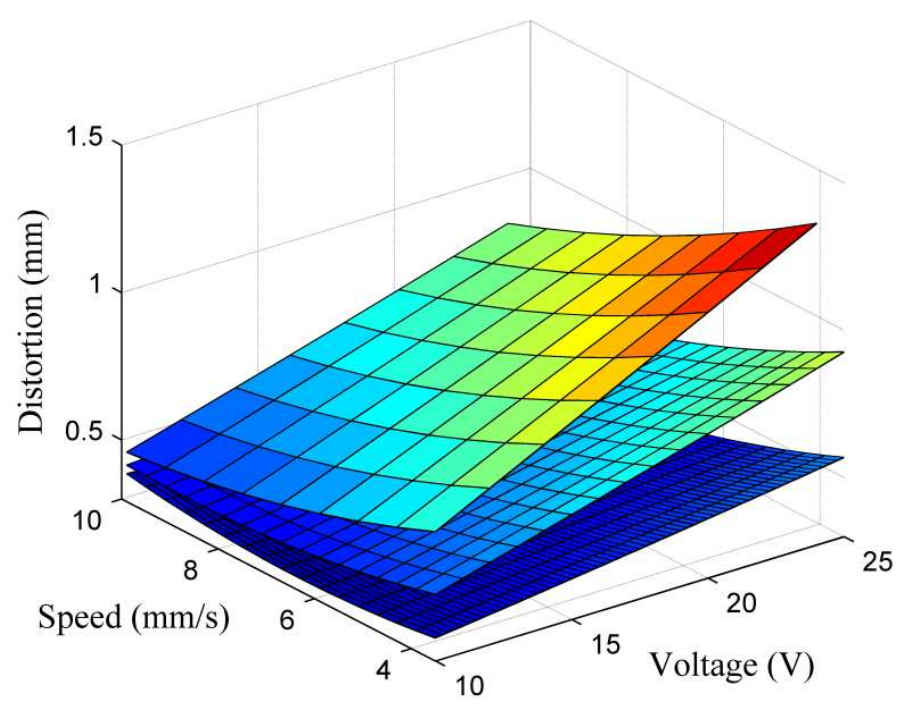

Figure 10. Interaction effect of voltage and speed on weld induced distortion. Coarse, medium and fine meshes represent $+1,0$ and -1 levels of other two variables respectively.

Figure 11 illustrates the interaction effect of current and speed on distortion by contour plots. Similar to previous plot, a coarse, medium and fine mesh size have been used in the figure to distinguish the $+1,0$ 
and -1 levels of other two variables respectively. As illustrated in Figure 11, with increase in current the distortion also increases while it decreases with increase in welding speed. Thereby, the optimum point is also likely to be located at a region where the current is at near lower bound while welding speed is at near upper bound. Also, it is observed that distortion value also increases with increase in other two variables.

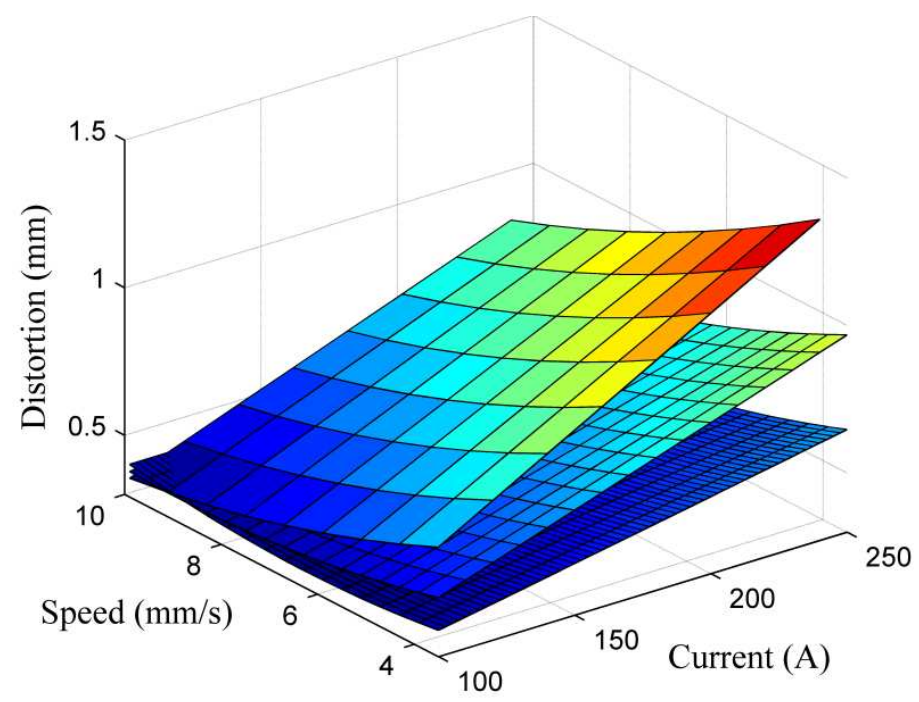

Figure 11. Interaction effect of current and speed on weld induced distortion. Coarse, medium and fine meshes represent $+1,0$ and -1 levels of other two variables respectively.

\section{Genetic Algorithm Based Optimization}

\subsection{Outline of Genetic Algorithms}

A generic GA can be considered as a controlled random walk; it efficiently exploits information from previous configurations to generate new configurations with improved performances expected. GA relies on stochastic search techniques based on the mechanism of natural selection and natural genetics. GA differing from conventional search techniques starts with an initial set of random solutions called population. Each individual in the population is called chromosome, representing a solution to the problem. The chromosomes evolve through successive iterations, called generations. During each generation, the chromosomes are evaluated using some measure of fitness or cost. To create the next generation, new chromosomes, called off-springs, are formed by either merging two chromosomes from current generation using a crossover operator or modifying a chromosome using a mutation operator. A new generation is formed by selecting, according to fitness/cost values, some of the parents and offsprings and rejecting others so as to keep the population size constant. Chromosomes with better fitness values have higher probability of getting selected. After several generations, the algorithm converges to the population containing the best chromosome, which is assumed to represent the optimum solution of the problem. The optimization algorithm runs until the maximum number of generations is reached or the cumulative change in the objective function value over five generations is less than or equal to predefined objective function tolerance. Interested readers may refer to the papers for more detailed working principle of GA (Goldberg, 1989).

\subsection{Optimization Problem Formulation}

The objective of this work is to minimize weld induced distortion. As such, it is considered as the criteria of optimization or objective function $\mathrm{F}(\mathrm{X})$. The distortion value will be determined using the 
corresponding RSM model developed in previous step. Welding speed (X1), arc voltage (X2), input current (X3) and welding direction (X4) are considered as design variables. Details of design variables and their bounds are shown in Table 4. Welding current ranged between 80 and 250 Ampere, arc voltage $(8-25$ Volt $)$, welding speed $(3.5-10 \mathrm{~mm} / \mathrm{s})$. The design variable associated with welding direction can take six numerical values to represent six possible welding directions as shown in Table 5.

Table 4. Description of design variables.

\begin{tabular}{ccccc}
\hline $\begin{array}{c}\text { Design } \\
\text { Variable }\end{array}$ & Definition & Unit & $\begin{array}{c}\text { Lower } \\
\text { Bound }\end{array}$ & $\begin{array}{c}\text { Upper } \\
\text { Bound }\end{array}$ \\
\hline X1 & Current & Amp & 100 & 250 \\
X2 & Voltage & Volt & 10 & 25 \\
X3 & Speed & $\mathrm{mm} / \mathrm{s}$ & 3.5 & 10 \\
X4 & Direction & - & 1 & 6 \\
\hline
\end{tabular}

Table 5. Definition of welding direction variable.

\begin{tabular}{cccc}
\hline Value & $\begin{array}{c}\text { Welding Direction } \\
\text { Symbol }\end{array}$ & $\begin{array}{c}\text { No. of } \\
\text { Robots }\end{array}$ & $\begin{array}{c}\text { Starting } \\
\text { Timing }\end{array}$ \\
\hline 1 & $\longrightarrow$ & 1 & Same \\
2 & $\longleftarrow$ & 1 & Same \\
3 & & 2 & Same \\
4 & $\longrightarrow-\longrightarrow$ & 2 & Same \\
5 & $-\cdots$ & 2 & Same \\
6 & $\longrightarrow$ & 2 & Same \\
\hline
\end{tabular}

To ensure a strong welded joint and good welding quality, it is important that the temperature in the welding zone is higher than or equal to melting temperatures of base metals and weld beads during welding. As such temperature constraints have been used to ensure good weld quality. During experimental design stage, temperatures at three different weld bead cross-sections are monitored in each welding simulation to ensure sufficient weld penetration as shown in Figure 12. Figure 12 (a) illustrates the three cross-sections (marked by red nodes) that were monitored for temperature constraint checking. Figure 12 (b) depicts the exact locations of the corresponding cross-sections in the simulation model. 
(b)

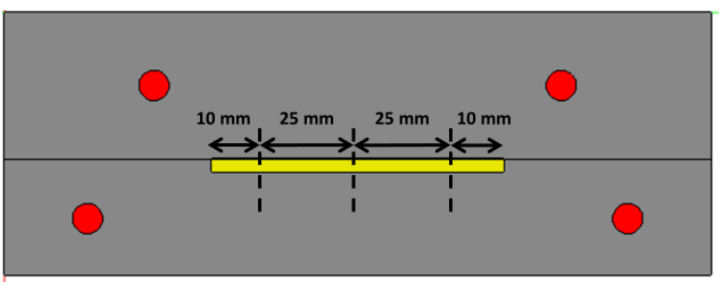

Figure 12. (a) Temperature constraint checking cross sections (marked by red nodes) and (b) exact location of cross sections.

Based on the weld penetration data, a RSM is built for considering the temperature effect. To incorporate this constraint violation into optimization algorithm, a penalty term is added to the objective function $\mathrm{F}(\mathrm{X})$ and the combined function is called augmented function. Whenever a constraint is violated, the penalty term is greater than zero, with the magnitude of the penalty being proportional to severity of constraint violations. In this work, the penalty term is proportional to surface area $\left(A_{c}\right)$ of weld bead that has temperature greater than bead material melting temperature during the welding. The augmented objective function definition including optimization constraints is defined as

$$
\varphi(\mathrm{x})= \begin{cases}\mathrm{F}(\mathrm{X}), & \mathrm{A}_{\mathrm{c}}>=1 \\ \mathrm{~F}(\mathrm{X})+100 *\left(1-\mathrm{A}_{\mathrm{c}}\right), & \mathrm{A}_{\mathrm{c}}<1\end{cases}
$$

As shown in Equation 2, a value of one for surface area $\left(A_{c}\right)$ of weld bead means that whole weld bead has received sufficient heat input and thereby good melting achieved. Consequently, the associated penalty term is nullified from objective function definition. However, when surface area $\left(\mathrm{A}_{c}\right)$ of weld bead is less than one, it indicates there is lack of sufficient heat input for which good weld penetration was not achieved. Obviously, such condition cannot be optimal even though the distortion value may be very small due to poor welding quality. The numeric value surface area $\left(\mathrm{A}_{c}\right)$ of weld bead is calculated from the regression equation derived for temperature constraint.

\subsection{Settings of $G A$}

The relevant GA parameters used in the optimization system are given in Table 6. The number of individuals in each iteration or population is 20 and maximum number of generations is 100 . A crossover rate of $80 \%$ is used and crossover operator is executed using scattered crossover function. Scattered crossover function creates a random binary vector and selects the genes where the vector is a 1 from the first parent, and the genes where the vector is a 0 from the second parent, and combines the genes to produce new chromosome. An adaptive mutation function has been used that randomly generates directions that are adaptive with respect to the last successful or unsuccessful generation. The mutation chooses a direction and step length that satisfies bounds and linear constraints.

Table 6. Genetic Algorithm Parameters used in RSM based optimization.

\begin{tabular}{cc}
\hline Parameter name & Value \\
\hline Population size & 20 \\
No of generations & 50 \\
Scaling fitness function & Rank \\
Parent selection & Stochastic uniform \\
Elite count & 2 \\
Crossover fraction & 0.8 \\
Function tolerance & $10 \mathrm{E}-06$ \\
Mutation function & Adaptive feasible \\
\hline
\end{tabular}




\subsection{Optimization Results}

After building the response surface models for distortion and temperature constraint evaluation, both models were linked with GA solver for welding optimization. The optimization results are illustrated in Table 7. Since GA is a deterministic algorithm, three trials were conducted to determine the optimum parameters for this case study. The best result has been found in third trial and the max distortion predicted is $0.39 \mathrm{~mm}$ with optimum values of considered design variables are $110.6 \mathrm{~V}, 24.41 \mathrm{~A}, 9.87$ $\mathrm{mm} / \mathrm{s}$ and 1 respectively. Figure 13 shows the optimization result convergence history with respect to the calculation generations or iterations for trial 3. The convergence history reveals that convergence was achieved with 45 generations with 920 function counts.

Table 7. Optimization results of GA based optimization approach.

\begin{tabular}{|c|c|c|c|c|c|}
\hline \multirow{2}{*}{$\begin{array}{c}\text { Trial } \\
\#\end{array}$} & \multicolumn{2}{|c|}{ Optimum values of design variables } & \multirow{2}{\text{Maximum}}{$\begin{array}{c}\text { Distortion } \\
\text { mm }\end{array}$} \\
\cline { 2 - 5 } & $\begin{array}{c}\text { X1, } \\
\text { Ampere }\end{array}$ & $\begin{array}{c}\mathrm{X} 2, \\
\text { Volt }\end{array}$ & $\begin{array}{c}\mathrm{X} 3, \\
\mathrm{~mm} / \mathrm{s}\end{array}$ & $\mathrm{X} 4$ & 0.44 \\
\hline 1 & 130.64 & 20.67 & 9.94 & 1 & 0.48 \\
\hline 2 & 148.06 & 18.24 & 9.64 & 1 & 0.39 \\
\hline 3 & 110.6 & 24.41 & 9.87 & 1 & \\
\hline
\end{tabular}

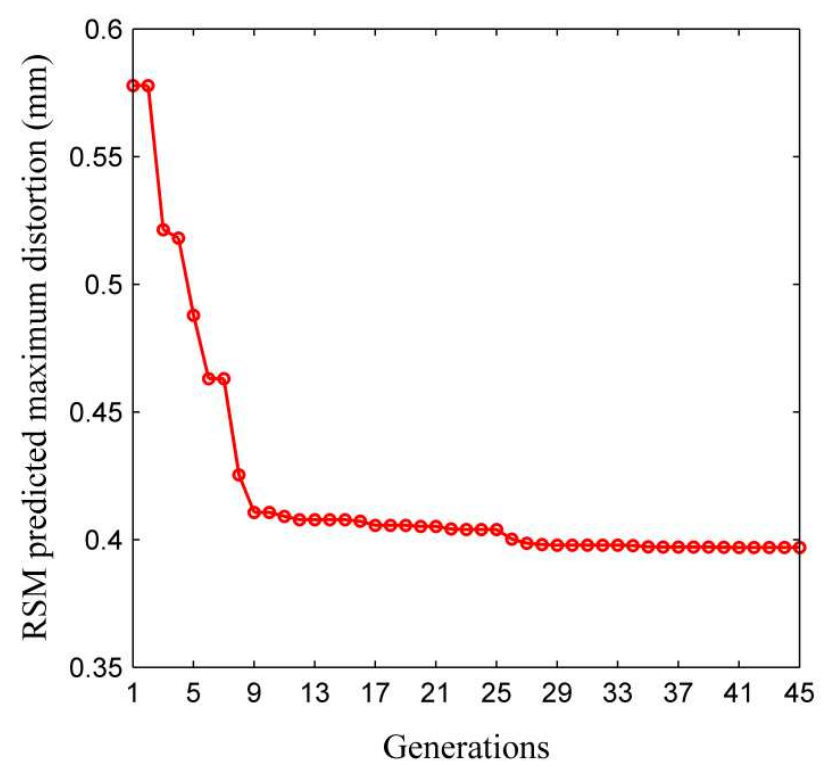

Figure 13. Result convergence history of GA based optimization approach.

\section{Conclusions}

Over the past decade, a tremendous growth has been observed in the use of CAE tools in manufacturing process design and analysis. By using CAE tools for numerical simulation of manufacturing processes, it is possible to predict the quality of finished product and identify potential defects during early design phase. Since a computer simulation is faster and cheaper than performing a real welding experiment, it reduces manufacturing cost and the time-to-market. Design optimization always requires a loop of designevaluate-redesign. Therefore, the ability to quickly and easily assess desired process dependent responses 


\section{Acknowledgements}

This research is based on the work supported by the US Department of Energy under Award Number DEEE0002323.Additional support provided by Center for Advanced Vehicular Systems (CAVS), Simufact Americas LLC and F.tech R\&D North America Inc. are acknowledged and greatly appreciated.

\section{References}

A, E.R.D.J., S, J.H.D., 2012. A review on optimization of welding process. Procedia Engineering 38, 544554.

Benyounis, K.Y., Olabi, A.G., 2008. Optimization of different welding processes using statistical and numerical approaches- A reference guide. Advances in Engineering Software 39, 483-496.

Brown, S.B., Song, H., 1992. Finite element simulation of welding of large structures. ASME Journal of Engineering for Industry 114, 441-451.

Camilleri, D., Gray, T.G.F., 2005. Computationally efficient welding distortion simulation techniques. Modeling and Simulation in Materials Science and Engineering 13, 1365-1382.

Correia, D.S., Goncalves, C.V., Cunha, S.S.d., Ferraresi, V.A., 2005. Comparison between genetic algorithms and response surface methodology in GMAW welding optimization. Journal of Material Processing Technology 160, 70-76.

Correia, D.S., Goncalves, C.V., Junior, S.S.C., Ferraresi, V.A., 2004. GMAW Welding Optimization Using Genetic Algorithms. Journal of Brazilian Society of Mechanical Science and Engineering XXVI, 28-33.

Deng, D., Liang, W., Murakawa, H., 2007a. Determination of welding deformation in fillet-welded joint by means of numerical simulation and comparison with experimental measurements. Journal of Material Processing Technology 183, 219-225.

Deng, D., Murakawa, H., 2008a. FEM prediction of buckling distortion induced by welding in thin plate panel structures. Computational Materials Science 43, 591-607. 
Deng, D., Murakawa, H., 2008b. Prediction of welding distortion and residual stress in a thin plate buttwelded joint. Computational Materials Science 43, 353-365.

Deng, D., Murakawa, H., Liang, W., 2007b. Numerical simulation of welding distortion in large structures. Computer Methods Applied Mechanical Engineering 196, 4613-4627.

Deng, D., Murakawa, H., Liang, W., 2008. Prediction of welding distortion in a curved plate structure by means of elastic finite element method. Journal of Material Processing Technology 203, 252-266.

Deng, D., Murakawa, H., Shibahara, M., 2010. Investigations on welding distortion in an asymmetrical curved block by means of numerical simulation technology and experimental method. Computational Materials Science 48, 187-194.

Goldak, J., Chakravarti, A., bibby, M., 1984. A new finite element model for welding heat source. Metallurgical Transactions B 15B, 299-305.

Goldak, J.A., Akhlaghi, M., 2005. Computational Welding Mechanics. Springer, New York.

Goldberg, D.E., 1989. Genetic Algorithms in Search, Optimization \& Machine Learning. AddisonWesley, Massachusetts.

Islam, M., Buijk, A., Rais-Rohani, M., Motoyama, K., 2014. Simulation-based numerical optimization of arc welding process for reduced distortion in welded structures. Finite Elements in Analysis and Design 84, 54-64.

Islam, M.R., Rohbrecht, J., Buijk, A., Namazi, E., Liu, B., Motoyama, K., 2013. Computational Optimization of Arc Welding Parameters Using Coupled Genetic Algorithms and Finite Element Method, Proceedings of the ASME 2013 International Mechanical Engineering Congress \& Exposition, San Diego, California, USA.

Jung, G.H., Tsai, C.L., 2004. Plasticity-based distortion analysis for fillet welded thin-plate T-joints. Welding Journal 83, 177-187.

Kadivar, M.H., Jafarpur, K., Baradaran, G.H., 2000. Optimizing welding sequence with genetic algorithm. Computational Mechanics 26, 514-519.

Kim, D., Kang, M., Rhee, S., 2005. Determination of Optimal Welding Conditions with a Controlled Random Search Procedure. Welding Journal 125-130.

Kim, D., Rhee, S., Park, H., 2002. Modeling and optimization of a GMA welding process by genetic algorithm and response surface methodology. International Journal of Production Research 40, 16991711.

Lindgren, L.-E., 2001a. Finite Element Modeling and Simulation of Welding Part 1: Increased Complexity. Journal of Thermal Stresses 24, 141-192.

Lindgren, L.-E., 2001b. Finite Element Modeling and Simulation of Welding. Part 3: Efficiency and Integration. Journal of Thermal Stresses 4, 305-334.

Lindgren, L.-E., 2007. Computational Welding Mechanics. Woodhead Publishing, Cambrige.

Lingren, L.E., 2001. Finite Element Modeling and Simulation of Welding. Part 2: Improved Material Modeling. Journal of Thermal Stresses 24, 195-231.

Michaleris, P., Debiccari, A., 1996. Predictive Technique for Buckling Analysis of Thin Section Panels due to Welding. Journal of Ship Production 12, 269-275.

Michaleris, P., Debiccari, A., 1997. Prediction of Welding Distortion. Welding Journal 76, 172-181.

Mollicone, P., Camilleri, D., Gray, T.G.F., Comlekci, T., 2006. Simple thermo-elastic-plastic models for welding distortion simulation. Journal of Material Processing Technology 176, 77-86.

Montgomery, D.C., 1997. Design and Analysis of Experiments, fourth ed. John Wiley \& Sons, New York.

Murakawa, H., Deng, D., Ma, N., Wang, J., 2011. Applications of inherent strain and interface element to simulation of welding deformation in thin plate structures. Computational Materials Science 51, 43-52.

Park, H.-S., Dang, X.-P., 2010. Structural Optimization based on CAD-CAE integration and metamodeling techniques. Computer-Aided Design 42, 889-902.

Song, J., Peters, J., Noor, A., Michaleris, P., 2003. Sensitivity analysis of the thermomechanical response of welded joints. International Journal of Solids and Structures 40, 4167-4181. 
Song, J., Shanghvi, J.Y., Michalers, P., 2004. Sensitivity analysis and optimization of thermo-elastoplastic processes with applications to welding side heater design. Computer Methods Applied Mechanical Engineering 193, 4541-4566.

Song, Y.-A., Park, S., Chae, S.-W., 2005. 3D welding and milling part II-optimization of the 3D welding process using an experimental design approach. International Journal of Machine Tools \& Manufacture 45, 1063-1069.

Sudhakaran, R., Murugan, V.V., Sivasakthivel, P.S., 2012. Optimization of process parameters to minimize angular distortion in gas tungsten arc welded statinless steel 202 grade plates using particle swarm optimization. Journal of Engineering Science and Technology 7, 195-208.

Tsai, C.L., Park, S.C., Cheng, W.T., 1999. Welding Distortion of a Thin-Plate Panel Structure. Welding Research Supplement, 156-165.

Tseng, H.-Y., 2006. Welding parameters optimization for economic design using neural approximation and genetic algorithm. International Journal of Advanced Manufacturing Technology 27, 897-901. 